\title{
MOLA PARCIAL CON FETO VIVO, COMPLICADO CON RESTRICCIÓN DE CRECIMIENTO INTRAUTERINO Y PREECLAMPSIA SEVERA. REPORTE DE CASO Y REVISIÓN DE LA LITERATURA
}

\section{Partial molar pregnancy with live fetus complicated by intrauterine growth restriction and severe preeclampsia. Case report and review of the} literature

Andrés Mauricio Camacho-Montaño, $M D^{1}$; Reinaldo Niño-Alba, $M D^{2}$

Recibido: 12 de diciembre de 2019 / Aceptado: 21 de julio de 2020

\section{RESUMEN}

Objetivo: reportar un caso de mola parcial con feto vivo y realizar una revisión de la literatura sobre las complicaciones maternas y fetales asociadas a esta condición.

Materiales y métodos: se presenta el reporte de un caso de mola parcial y feto vivo de 33 semanas, complicado por restricción de crecimiento intrauterino, oligoamnios y preeclampsia severa. Después de seguimiento del recién nacido a un año reportamos un resultado satisfactorio materno-fetal. Se realiza una búsqueda de la literatura en Medline vía PubMed, LILACS, OVID, Uptodate y Google Scholar, con los siguientes términos MESH: "hydatidiform mole", "partial mole”, "live fetus", "coexisting live

* Correspondencia: Andrés Mauricio Camacho Montaño, calle 100 \# 48f-17. Bogotá (Colombia). Camacho.andresm@gmail.com

1 Médico cirujano, especialista ginecología y obstetricia. Subred Servicios de Salud Centro Oriente, Bogotá (Colombia).

2 Médico cirujano, Universidad Nacional de Colombia; especialista ginecología y obstetricia, Universidad Nacional de Colombia, Bogotá (Colombia). fetus". Se seleccionaron estudios de series de caso y reportes de caso de gestantes con coexistencia de mola parcial y feto vivo al momento del diagnóstico, y se extrajo información sobre el pronóstico materno-fetal.

Resultados: se identificaron inicialmente 129 títulos relacionados, de los cuales 29 cumplieron los criterios de inclusión, 4 artículos fueron excluidos por no obtener acceso al texto completo. Se analizaron 31 casos reportados, 9 casos terminaron en aborto, 8 terminaron en óbito o muerte perinatal y 14 (45\%) casos terminaron con un recién nacido vivo. La complicación materna más frecuente fue preeclampsia, en 6 (19,35\%) casos.

Conclusión: la coexistencia de mola parcial con feto vivo presenta un riesgo alto de resultado perinatal adverso y preeclampsia. Se requiere más información sobre esta rara condición para determinar de mejor manera posibles intervenciones en los casos de fetos euploides y dar una adecuada asesoría en la práctica clínica, por lo que es impor- 
tante el reporte de estos casos para tener suficiente evidencia sobre el comportamiento natural de la enfermedad.

Palabras clave: mola parcial; feto vivo; preeclampsia; restricción de crecimiento intrauterino.

\section{ABSTRACT}

Objective: To report the case of a partial molar pregnancy with live fetus and conduct a review of the literature regarding maternal and fetal complications associated to this condition.

Materials and methods: Case report of a partial mole with a 33 weeks live fetus complicated by intrauterine growth restriction, oligohydramnios and severe preeclampsia. We report satisfactory maternal and neonatal outcomes and 1-year follow-up. A search was conducted in the Medline via Pubmed, LILACS, Ovid, Uptodate and Google Scholar databases using the following MESH terms: hydatidiform mole, partial mole, live fetus, coexisting live fetus. Case series and case reports of pregnant women with coexisting partial mole and live fetus at the time of diagnosis were selected and information regarding maternal and fetal prognosis was extracted.

Results: Initially, 129 related titles were identified. Of these, 29 met the inclusion criteria, and 4 articles were excluded due to failed access to the full text. Overall, 31 reported cases were included; 9 ended in miscarriage, 8 in fetal demise or perinatal death, and 14 (45\%) resulted in a live neonate. The most frequent maternal complication was preeclampsia in $6(19.35 \%)$ cases.

Conclusion: The coexistence of a partial mole with a live fetus poses a high risk of adverse perinatal outcomes and preeclampsia. The volume of information regarding this rare condition must be increased in order to better determine potential interventions in cases of euploid fetuses and to provide adequate counseling in clinical practice. Therefore, reporting these cases is important to build sufficient evidence about the natural course of this condition.
Key words: Partial mole; live fetus; preeclampsia; intrauterine growth restriction.

\section{INTRODUCCIÓN}

La mola hidatiforme es una de las formas de presentación de la enfermedad trofoblástica gestacional, junto con el coriocarcinoma, la mola invasora y el tumor trofoblástico del sitio placentario (1). La mola hidatiforme es una patología placentaria caracterizada por una hiperproliferación de las vellosidades placentarias y degeneración hidrópica (2). La mola se clasifica como completa o parcial (3).

La mola hidatiforme completa se caracteriza por una alteración placentaria que tiene un conjunto diploide de cromosomas de origen paterno, sin presencia de tejido fetal (4). Desde el punto de vista citogenético, se desarrolla después de que un óvulo, cuyo material genético se ha perdido o dañando, es fertilizado por uno o dos espermatozoides, lo que resulta en un zigoto diploide de origen androgenético (3). El cariotipo es $46 \mathrm{XX}$ en el $90 \%$ de los casos y $46 \mathrm{XY}$ en el $10 \%$ de los casos (1). La mola parcial se caracteriza por la alteración placentaria más la presencia de tejido fetal (5). Candelier indica que, según la teoría de Golubovsky, se produce por la fertilización de un óvulo normal por dos espermatozoides, que dan origen a una triploidía en la mayoría de los casos, por lo que usualmente es un feto polimalformado que termina en aborto (2). Sin embargo, hay casos reportados de fetos euploides que pueden llegar a embarazos viables (6).

La incidencia de mola completa varía en diferentes regiones; la más alta está en el sudeste asiático, con una incidencia de 13 por 1.000 embarazos, y la más baja en Suramérica, con una incidencia de 0,3 por 1.000 embarazos (3). La incidencia de mola parcial es de 3 en 1.000 (1) y la incidencia de la coexistencia de feto vivo con mola parcial es de 1 en 22.000 a 1 en $100.000(6,7)$.

La coexistencia de mola y feto vivo puede deberse a la presencia de un embarazo múltiple, donde uno de ellos es normal, con feto y placenta normales, y el otro es un embarazo molar, ya sea 
una mola completa o parcial. La otra posibilidad, mucho menos frecuente, como se mencionó, es que se trate de una mola parcial con feto vivo eupliode, de la cual solo hay pocos casos reportados en la literatura $(6,8)$.

Existen tres revisiones importantes de coexistencia de embarazo molar y feto vivo; la primera realizada por Vejerslev en 1991, que analiza la literatura desde 1903 hasta 1989, y reporta 113 casos de mola completa con coexistencia de feto vivo; de estos, 3 casos correspondieron a mola parcial con feto vivo (6). La segunda revisión es la realizada por Wee y Jauniaux en 2005, donde reportan 174 casos de mola completa y feto vivo (9). La tercera la realiza Lin et al., en 2017 en 2017, que reporta una serie de 72 casos de embarazos múltiples con mola completa y feto vivo, y se revisan 9 artículos que en conjunto suman 173 casos más (10).

El diagnóstico prenatal de embarazo molar y feto vivo se basa en niveles elevados beta HCG y en los hallazgos ultrasonográficos (9). Se ha descrito que el ultrasonido tiene una sensibilidad del $44 \%$ y una especificidad del $74 \%$ en el primer trimestre, y que como diagnóstico diferencial está el aborto incompleto y el huevo anembrionado (11).

Respecto al manejo de la mola con feto vivo, algunos autores recomiendan el uso de la amniocentesis para determinación del cariotipo fetal $(6,9)$. En el caso de embarazo temprano, Bruchim recomienda su interrupción cuando el diagnóstico se hace en la primera mitad del embarazo, sin embargo, este autor aconseja un manejo expectante si no se detectan malformaciones y el cariotipo es normal (12). Es poca la información sobre el manejo de los fetos vivos luego de la semana 22 hasta el parto. Lin reporta la necesidad de terminación del embarazo en $40 \%$ de los casos por complicaciones médicas (10). Posterior al parto se puede presentar enfermedad trofoblástica persistente, más frecuentemente en mola completa y feto vivo (21\%) (13), pero también descrita en el $1 \%$ en los casos de mola parcial (2). Por esta razón, se hace seguimiento semanal de la gonadotrofina coriónica humana fracción beta (B GCH) una vez terminada la gestación hasta que sea negativa (14).

Se ha descrito que la presencia de un embarazo gemelar con mola y feto vivo se asocia con un aumento de complicaciones maternas tales como preeclampsia en un 20 a $30 \%(6,9)$, sangrado vaginal en 46\%, hiperémesis gravídica en $17 \%$ (6). Desde el punto de vista fetal, se informa amplia variabilidad en los datos de sobrevida del recién nacido que van desde el $25 \%$ (9) hasta cerca del $60 \%(6,10)$. En estos recién nacidos se reporta una importante presencia de restricción de crecimiento intrauterino y parto pretérmino (6).

Como se observa, hay varias publicaciones sobre mola completa y feto vivo, sin embargo, la información es limitada en lo que corresponde específicamente a casos de mola parcial con feto vivo, en cuanto al tratamiento de acuerdo con la edad gestacional del diagnóstico, y el pronóstico fetal y materno, por lo que nuestro objetivo es hacer un reporte de caso y realizar una revisión de la literatura, enfocándonos en el resultado materno y fetal de esta condición.

\section{REPORTE DE CASO}

Presentamos el caso de una mujer de 19 años, primigestante, con gestación de 33 semanas, residente en área rural del departamento del Meta, en la región de los Llanos Orientales de Colombia, con cuadro clínico de una semana de evolución de edema generalizado y cifras de presión arterial de 154/94, por lo que es remitida a la ciudad de Bogotá con diagnóstico de preeclampsia. Antecedentes médico-quirúrgicos negativos y antecedente familiar de madre hipertensa.

Había iniciado su control prenatal desde la semana 16, cuando se registró un peso de $53 \mathrm{~kg}$ y talla $160 \mathrm{~cm}$. Laboratorios obligatorios del control prenatal normales y hormona tiro-estimulante (en inglés TSH) dentro de límites normales. Traía tres ultrasonidos de su control prenatal. Ultrasonido 
semana 16: feto con anatomía normal, líquido y placenta normales. Ultrasonido semana 22: feto con quiste hepático, líquido amniótico normal y lesión placentaria sospechosa de acretismo placentario. Ultrasonido de semana 32: restricción de crecimiento intrauterino: biometría para 28 semanas, oligoamnios (ILA $=4 \mathrm{~cm}$ ). Placenta con sospecha de acretismo placentario.

En marzo de 2018, la paciente ingresa a la sede materno-infantil del hospital La Victoria, institución de tercer nivel ubicada en la ciudad de Bogotá, que atiende pacientes pertenecientes al régimen subsidiado por el Estado en el Sistema General de Seguridad Social en Colombia. La paciente ingresa consiente, alerta, hidratada, peso 61 k, con presión arterial 150/100, altura uterina de $28 \mathrm{~cm}$, edema grado II de miembros inferiores y resto del examen físico sin alteraciones. Se solicitan laboratorios y ultrasonido de detalle.

El ultrasonido obstétrico de detalle anatómico arroja los siguientes resultados: peso estimado fetal, $830 \mathrm{~g}$; crecimiento menor del percentil 3 para la edad gestacional. En la valoración de la placenta se encuentra una imagen de $90 \times 167 \mathrm{~mm}$, con múltiples imágenes hipoecoicas en patrón de panal de abejas, sugestiva de enfermedad trofoblástica gestacional (figuras 1 y 2). La valoración de líquido amniótico confirma oligoamnios (ILA $=3 \mathrm{~cm}$ ). En la evaluación Doppler se descarta acretismo placentario. El Doppler de hemodinamia fetal muestra aumento de resistencia en arteria umbilical. Resistencias normales en arteria cerebral media y ductus venoso. La valoración fetal evidencia feto de sexo femenino, con lesión focal hepática en lóbulo derecho de $25 \times 26 \mathrm{~mm}$, hipoecoica irregular, con septos pequeños y sugestiva de hamartoma mesenquimatoso hepático (figura 3); resto de la anatomía fetal normal. Se realiza una impresión diagnóstica de gestación de 33 semanas, preeclampsia sin criterios de severidad, enfermedad trofoblástica con coexistencia de feto vivo, restricción de crecimiento intrauterino, oligoamnios, quiste hepático fetal:

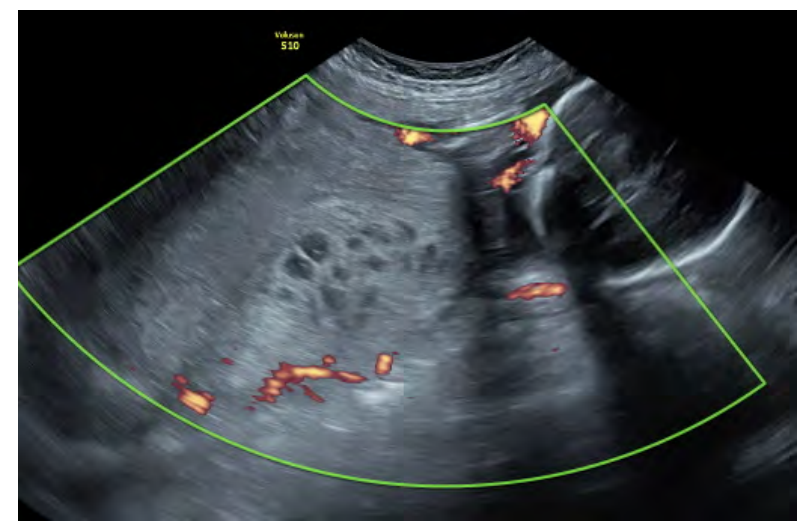

Figura 1. Imagen de ultrasonido, con zona de placenta normal y zona de placenta alterada con degeneración hidrópica. Paciente con embarazo feto vivo y mola parcial, Bogotá (Colombia), 2018

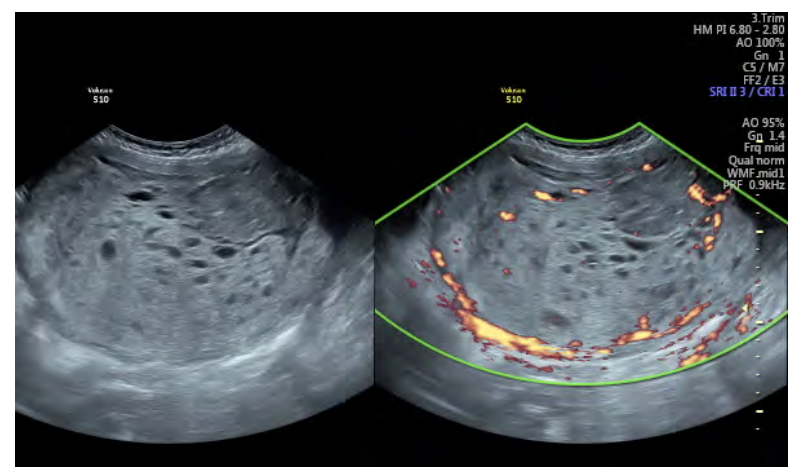

Figura 2. Acercamiento a la placenta con patrón clásico de panal de abejas, sugestivo de enfermedad trofoblástica gestacional. Paciente con embarazo feto vivo y mola parcial, Bogotá (Colombia), 2018

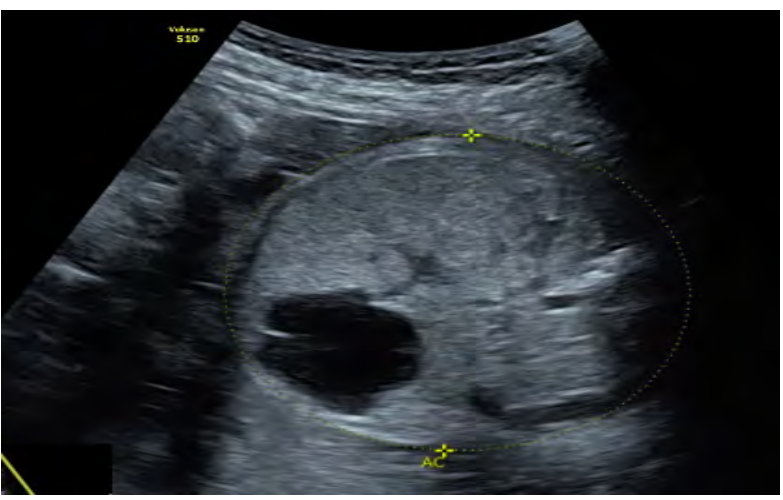

Figura 3. Imagen quística intrahepática fetal, sugestiva de hamartoma. Paciente con embarazo feto vivo y mola parcial, Bogotá (Colombia), 2018 
sospecha de hamartoma hepático. En cuanto a los laboratorios, el hemograma informó: hemoglobina, $14 \mathrm{~g} / \mathrm{dl}$; hematocrito, $41 \%$; leucocitos, $12.300 / \mathrm{mm}^{3}$; plaquetas, $166.000 / \mathrm{mm}^{3}$; creatinina, 0,6 mg/dl; transaminasas: AST, $32 \mathrm{UI}$, ALT, $34 \mathrm{UI}$; proteinuria de 24 horas, $400 \mathrm{mg}$. No se tomó gonadotropina por lo avanzado de la gestación.

La paciente presenta inicio de trabajo de parto espontáneo 6 horas después del ingreso, con 33 semanas de gestación, el cual se conduce sin complicaciones; luego de 6 horas de trabajo de parto se atiende parto vaginal con recién nacido de sexo femenino de 900 g, APGAR 8-9-10, al minuto, 5 y 10 minutos. Placenta tipo Shultze completa. Luego del alumbramiento, en sala de partos presenta hemorragia en el puerperio inmediato secundario a atonía uterina, con frecuencia cardiaca de 114 latidos/ min, presión arterial de 148-95 mm/Hg. Se activa protocolo de hemorragia posparto, denominado en Colombia código rojo (15); la hemorragia cede con manejo farmacológico con oxitocina. Se realiza revisión uterina bajo anestesia, se transfunden $2 \mathrm{U}$ de glóbulos rojos. Reporte de laboratorios tomados durante la aplicación del código rojo: hemoglobina, $11 \mathrm{~g} / \mathrm{dl}$; hematocrito, 34\%; plaquetas, 183,000/ mm³; fibrinógeno, $775 \mathrm{mg} / \mathrm{dl}$; dímero, D $30 \mathrm{ng} / \mathrm{ml}$.

En el puerperio temprano presenta cifras de presión arterial con criterios de preeclampsia severa $(184 / 111 \mathrm{~mm} / \mathrm{Hg})$ que no se controlan con la administración de nifedipina y clonidina, por lo que se traslada a unidad de cuidados intensivos y se maneja con labetalol en infusión continua a $1 \mathrm{mg} /$ min; refiere pérdida transitoria de la visión por lo que se realiza tomografía axial computarizada (TAC) cerebral, reportada como normal. Se identifica aumento de proteinuria de $6,3 \mathrm{~g}$ en orina de $24 \mathrm{~h}$, otras pruebas para el diagnóstico de severidad de preeclampsia en límites normales. Luego de $72 \mathrm{~h}$ se da egreso de la unidad de cuidados intensivos y después de 15 días se da salida de hospitalización, con fórmula de losartan $150 \mathrm{mg} /$ día, nifedipina 90 $\mathrm{mg}$ /día y clonidina $900 \mathrm{mcg}$ /día. El reporte final de patología de placenta muestra placenta monocorial monoamniótica de $550 \mathrm{~g}$, en la cual se reconocen vellosidades coriales con degeneración hidrópica, reportada como enfermedad trofoblástica gestacional tipo mola parcial.

La recién nacida recibió dos dosis de surfactante y requirió soporte ventilatorio por 5 días. Presentó sepsis neonatal por Kluyvera ascorbata BLEE, manejada con ertapenem, de evolución positiva. Durante la hospitalización se realizaron los siguientes estudios: ecografía de abdomen total que muestra imagen quística compatible con hamartoma, ecografía transfontanelar normal, ecocardiograma normal, ecografía renal normal y cariotipo 46XX normal. Es valorada por cirugía pediátrica quien considera control ambulatorio. Estuvo hospitalizada por 62 días y se dio salida con suplencia de oxígeno domiciliario por displasia pulmonar moderada. Seguimiento de la madre y la niña a un año, sin evidencia de enfermedad trofoblástica persistente, con b-HCG negativa y sin requerimiento farmacológico antihipertensivo. La niña tuvo una evolución satisfactoria.

Consideraciones éticas. La paciente fue informada del diagnóstico, firmó consentimiento informado para cada procedimiento realizado y firmó un consentimiento expreso para la publicación de este caso. Se tomaron las precauciones para garantizar la confidencialidad de la información y el anonimato de la paciente; el registro fotográfico fue tomado por los autores.

\section{MATERIALES Y MÉTODOS}

Para responder a la pregunta sobre las complicaciones maternas y fetales asociadas a los casos de mola parcial y feto vivo, se realizó una búsqueda de la literatura en las bases de datos: Medline vía PubMed, google scholar y LILACS, con los siguientes términos MESH: "hydatidiform mole", "partial mole", "live fetus", "coexisting live fetus", en inglés y español, sin límite de tiempo. Criterios de inclusión de los estudios: reportes o series de casos; tipo de población: gestantes con diagnóstico de mola incompleta con 
feto vivo. Se utilizó el método de "bola de nieve" y se realizó búsqueda a partir de las referencias citadas en cada artículo. La selección y extracción de los datos y revisión fue realizada por un autor y verificada por el otro. Se tomó información sobre las siguientes variables: país donde se presentó el caso, edad materna, edad gestacional, diagnóstico, método de diagnóstico, tipo de manejo (expectante, suspensión del embarazo), tipo de parto (cesárea o parto vaginal), resultado fetal (óbito fetal, recién nacido vivo, retardo de crecimiento intrauterino, malformaciones congénitas), complicaciones maternas (anteparto, intraparto o posparto), complicaciones del recién nacido. Los resultados se presentan de manera narrativa.

\section{RESULTADOS}

Inicialmente se identificaron 128 títulos y resúmenes; de estos, un total de 29 estudios cumplieron los criterios de inclusión, de los cuales en 25 se tuvo acceso al texto completo y en 4 solo se tuvo acceso al resumen, motivo por el cual fueron descartados de la revisión (16-19). Los 25 estudios incluidos correspondieron a 25 reportes de casos.

En total se encontraron 31 casos de la coexistencia de mola incompleta y feto vivo.

Sitio del estudio: un total de 5 estudios fueron realizados en Estados Unidos (13,20-23), tres en India (24-26), dos en México (27,28), dos en China $(29,30)$ dos de Turquía $(34,41)$ y los otros reportes fueron en: Australia (31), Holanda (32), Inglaterra (33), Pakistán (35), Nepal (36), Polonia (42), Bangladesh (37), Chile (38), Malasia (39), Italia (43) y Canadá (40).

La edad materna se encontró en el rango de 17 a 29 años en 25 casos (13,22-29,32-37,39-42), de 30 a 39 años en 6 casos $(20,21,23,30,31,43)$ y mayores o iguales a 40 años en ningún caso. Respecto a la edad gestacional al momento del diagnóstico fue antes de las 22 semanas en 17 casos (20-23,25,28,30,33$35,40)$, de 23 a 27 semanas en 4 casos $(29,32,38,41)$, de 28 a 37 semanas 6 casos $(24,27,36,37,39)$ y 37 semanas o más en 4 casos $(13,26,31)$. El método de diagnóstico fue ultrasonido prenatal en 21 casos $(20,21,23-25,27,28,30,32-35,37,40,42-43)$, y en el alumbramiento o por patología, 10 casos $(13,26,29,31,36,38,39,41,43)$. Se ofreció terminación del embarazo en 4 casos $(20,23)$.

La edad gestacional al momento de la terminación del embarazo osciló entre las 15 y las 40 semanas: de 22 semanas o menos, 9 casos $(20,22,23,28,40)$; de 23 a 27 semanas, 4 casos $(29,32,34,38)$; de 28 a 37 semanas, 13 casos $(21,24-25$ - 26-28,30,33,36,39,41,4243) y de 38 a 42 semanas, 5 casos $(13,26,31,35,37)$. Respecto al tipo de parto: parto por cesárea, 12 casos $(8,21,26,27,30-34,39,42,43)$, parto vaginal, 10 casos $(13,25,28,29,35-37,41,42)$.

Resultado fetal: 9 casos terminaron en aborto $(20,22,23,28,40)$; 8 casos presentaron óbito o muerte neonatal temprana $(24,28,29,32,34,38,39,41)$. Restricción de crecimiento intrauterino, 6 casos (24,25,27,37,39,43); malformaciones fetales, 2 casos; un caso de Dandy Walker (26) y un caso con mielomeningocele (38). En 14 casos se obtuvo un feto vivo, viable $(13,21,25,26,27,30,31,33,35-37,42,43)$. En cuanto a las complicaciones maternas reportadas: preeclampsia en 6 casos $(6,23,28,31,34,40)$, un caso de hemorragia posparto que requirió histerectomía (27), enfermedad trofoblástica persistente en 3 casos $(4,23)$, un caso con crisis hipertiroidea (41); no se presentaron casos de sepsis o mortalidad materna.

La información de los reportes de caso se observa en la tabla 1.

\section{CONCLUSIÓN}

La coexistencia de mola parcial con feto vivo presenta un riesgo alto de resultado perinatal adverso y preeclampsia. Se requiere incrementar el volumen de información sobre esta rara condición para determinar posibles intervenciones en los casos de fetos euploides y dar una adecuada asesoría en la práctica clínica, por lo que es importante el reporte de estos casos a fin de construir la suficiente evidencia sobre el comportamiento natural de la enfermedad. 
Tabla 1.

Estudios de reporte de caso incluidos en la revisión de la literatura de mola parcial y feto vivo

\begin{tabular}{|c|c|c|c|c|c|c|c|}
\hline Autor & País & $\begin{array}{c}\text { Edad de } \\
\text { la madre } \\
\text { (años) }\end{array}$ & $\begin{array}{c}\text { EG } \\
\text { (semanas) }\end{array}$ & $\begin{array}{c}\text { Peso RN } \\
\text { (g) }\end{array}$ & $\begin{array}{l}\text { Sexo } \\
\text { RN }\end{array}$ & $\begin{array}{l}\text { Complicación } \\
\text { fetal }\end{array}$ & $\begin{array}{c}\text { Complicación } \\
\text { materna }\end{array}$ \\
\hline $\begin{array}{l}\text { Jones et al., } \\
1975 \text { (13) }\end{array}$ & USA & 17 & 40 & 2900 & Fem. & Ninguna & Ninguna \\
\hline $\begin{array}{l}\text { Hartfiel eet } \\
\text { al., } 1983 \text { (20) }\end{array}$ & Australia & 36 & 39 & 2700 & Masc. & Ninguna & Preeclampsia \\
\hline $\begin{array}{l}\text { Crooij et al., } \\
1985 \text { (21) }\end{array}$ & Holanda & 23 & 27 & 980 & Fem & $\begin{array}{l}\text { Muerte neonatal } \\
\text { temprana }\end{array}$ & Ninguna \\
\hline $\begin{array}{l}\text { Deaton et al., } \\
1989 \text { (22) }\end{array}$ & USA & 33 & 15 & ND & Fem. & Aborto & Ninguna \\
\hline $\begin{array}{l}\text { Thomas et al., } \\
1987 \text { (23) }\end{array}$ & Inglaterra & 23 & 29 & 1150 & Fem. & Ninguna & Ninguna \\
\hline $\begin{array}{l}\text { Cheung et al., } \\
1992 \text { (24) }\end{array}$ & China & 24 & 27 & 970 & Fem. & Muerte neonatal & $\begin{array}{l}\text { Enfermedad } \\
\text { trofoblástica } \\
\text { persistente }\end{array}$ \\
\hline $\begin{array}{l}\text { Hsieh et al., } \\
1999 \text { (25) }\end{array}$ & USA & 30 & 32 & 1551 & Fem. & Anemia & Ninguna \\
\hline $\begin{array}{l}\text { Lembert } \\
\text { et al., } 2000 \\
\text { (26) }\end{array}$ & Turquía & 28 & 23 & 560 & Masc. & $\begin{array}{l}\text { Óbito. Malforma- } \\
\text { ción fetal }\end{array}$ & preeclampsia \\
\hline $\begin{array}{l}\text { Parveen et al., } \\
2004 \text { (27) }\end{array}$ & Pakistán & 23 & 39 & 2700 & Fem. & Ninguna & Ninguna \\
\hline $\begin{array}{l}\text { Guven et al., } \\
2007 \text { (28) }\end{array}$ & Turquía & 21 & 28 & 800 & Fem. & Óbito & $\begin{array}{c}\text { Crisis } \\
\text { hipertiroidea }\end{array}$ \\
\hline $\begin{array}{l}\text { Tamrakar } \\
\text { et al., } 2011 \\
\text { (29) }\end{array}$ & Nepal & 26 & 32 & 1100 & Masc. & Ninguna & Ninguna \\
\hline $\begin{array}{l}\text { Shobhau } \\
\text { et al., } \\
2011 \text { (30) }\end{array}$ & India & 21 & 37 & 1500 & Masc. & Óbito RCIU & Ninguna \\
\hline $\begin{array}{l}\text { Sak et al., } \\
2012 \text { (31) }\end{array}$ & Polonia & 28 & 37 & 2800 & Fem. & Ninguna & Ninguna. \\
\hline $\begin{array}{l}\text { Rathod et al., } \\
2014 \text { (32) }\end{array}$ & India & 23 & 34 & 1400 & Fem. & RCIU & Ninguna \\
\hline $\begin{array}{l}\text { Ara et al., } \\
2016(33)\end{array}$ & Bangladésh & 26 & 40 & 1500 & Masc. & RCIU & Ninguna \\
\hline $\begin{array}{l}\text { Florez } 2016 \\
\text { (34) }\end{array}$ & Chile & 21 & 25 & 610 & Masc. & $\begin{array}{l}\text { Óbito polimalfor- } \\
\text { mado }\end{array}$ & No \\
\hline $\begin{array}{l}\text { García et al., } \\
2017 \text { (35) }\end{array}$ & México & 27 & 33 & 1417 & Fem. & RCIU & Hemorragia \\
\hline
\end{tabular}




\begin{tabular}{|c|c|c|c|c|c|c|c|}
\hline Autor & País & $\begin{array}{c}\text { Edad de } \\
\text { la madre } \\
\text { (años) }\end{array}$ & $\begin{array}{c}\text { EG } \\
\text { (semanas) }\end{array}$ & $\begin{array}{c}\text { Peso RN } \\
\text { (g) }\end{array}$ & $\begin{array}{l}\text { Sexo } \\
\text { RN }\end{array}$ & $\begin{array}{c}\text { Complicación } \\
\text { fetal }\end{array}$ & $\begin{array}{c}\text { Complicación } \\
\text { materna }\end{array}$ \\
\hline $\begin{array}{l}\text { Atuck et al., } \\
2018 \text { (36) }\end{array}$ & Malasia & 21 & 28 & 990 & Fem. & $\begin{array}{c}\text { RCIU. Muerte } \\
\text { neonatal temprana }\end{array}$ & Preeclampsia \\
\hline $\begin{array}{l}\text { Zeng et al., } \\
2019 \text { (37) }\end{array}$ & China & 32 & 29 & 1050 & Fem. & Ninguna & Ninguna \\
\hline $\begin{array}{l}\text { De Francicis } \\
2019 \text { et al., } \\
(38)\end{array}$ & Italia & 37 & 31 & 880 & Masc. & RCIU & Ninguna \\
\hline $\begin{array}{l}\text { Hassan et al., } \\
2018 \text { (39) }\end{array}$ & USA & 25 & 20 & 200 & Masc. & Aborto & Ninguna \\
\hline \multirow{2}{*}{$\begin{array}{l}\text { Saurbrei } \\
\text { et al., } \\
1980 \text { (40) }\end{array}$} & \multirow[t]{2}{*}{ Canadá } & 27 & 21 & 225 & Fem. & Aborto & Preeclampsia \\
\hline & & 22 & 20 & 450 & Masc. & Aborto & Preeclampsia \\
\hline \multirow[t]{3}{*}{$\begin{array}{l}\text { Teng et al., } \\
1984 \text { (41) }\end{array}$} & \multirow[t]{3}{*}{ USA } & 30 & 15 & 400 & Fem. & Aborto & $\begin{array}{l}\text { Enfermedad } \\
\text { trofoblástica } \\
\text { persistente }\end{array}$ \\
\hline & & 22 & 15 & 300 & Masc. & Aborto & $\begin{array}{l}\text { Enfermedad } \\
\text { trofoblástica } \\
\text { persistente }\end{array}$ \\
\hline & & 24 & 20 & 300 & Fem & Aborto & Preeclampsia \\
\hline \multirow{3}{*}{$\begin{array}{l}\text { Juárez et al., } \\
2009 \text { (42) }\end{array}$} & \multirow[t]{3}{*}{ México } & 24 & 20 & 300 & Fem & Aborto & Preeclampsia \\
\hline & & 21 & 31 & 1050 & Fem. & Muerte perinatal & Ninguna \\
\hline & & 22 & 19 & 480 & Masc. & Aborto & Ninguna \\
\hline \multirow{2}{*}{$\begin{array}{l}\text { Dhingra } \\
\text { et al., } \\
2009 \text { (43) }\end{array}$} & \multirow[t]{2}{*}{ India } & 28 & 38 & 2300 & Fem. & Ninguna & Ninguna \\
\hline & & 22 & 34 & 1400 & Fem. & Ninguna & Ninguna \\
\hline
\end{tabular}

EG: edad gestacional.

$\mathrm{RN}$ : recién nacido.

RCIU: retardo de crecimiento fetal intrauterino.

\section{CONTRIBUCIÓN DE LOS AUTORES}

Los dos autores participaron por igual en la elaboración del documento, desde su concepción y diseño hasta la adquisición de la información, revisión del contenido intelectual y aprobación de la versión enviada a proceso editorial.

\section{REFERENCIAS}

1. Seckl MJ, Sebire NJ, Berkowitz RS. Gestational trophoblastic disease. Lancet. 2010;376(9742):717-29. https://doi.org/10.1016/S0140-6736(10)60280-2
2. Candelier JJ. The hydatidiform mole. Cell Adh Migr. 2016;10(1-2):226-35. https://doi.org/10.1080/1933 6918.2015 .1093275

3. Hui P, Buza N, Murphy KM, Ronnett BM. Hydatidiform moles: Genetic basis and precision diagnosis. Annu Rev Pathol. 2017;12:449-85. https://doi. org/10.1146/annurev-pathol-052016-100237

4. Kajii T, Ohama K. Androgenetic origin of hydatidiform mole. Nature. 1977;268(5621):633-4. https://doi. org $/ 10.1038 / 268633 \mathrm{a} 0$ 
5. Jauniaux E, Brown R, Rodeck C, Nicolaides KH. Prenatal diagnosis of triploidy during the second trimester of pregnancy. Obstet Gynecol. 1996;88(6):983-9. https://doi.org/10.1016/S0029-7844(96)00330-4

6. Vejerslev LO. Clinical management and diagnostic possibilities in hydatidiform mole with coexistent fetus. Obstet Gynecol Surv. 1991;46(9):577-88. https://doi. org/10.1097/00006254-199109000-00001

7. Steller MA, Genest DR, Bernstein MR, Lage JM, Goldstein DP, Berkowitz RS. Clinical features of multiple conception with partial or complete molar pregnancy and coexisting fetuses. J Reprod Med. 1994;39(3):147-54.

8. Hsieh CC, Hsieh TT, Hsueh C, Kuo DM, Lo LM, Hung TH. Delivery of a severely anaemic fetus after partial molar pregnancy: Clinical and ultrasonographic findings. Hum Reprod. 1999;14(4):1122-6. https:// doi.org/10.1093/humrep/14.4.1122

9. Wee L, Jauniaux E. Prenatal diagnosis and management of twin pregnancies complicated by a co-existing molar pregnancy. Prenat Diagn. 2005;25(9):772-6. https:// doi.org/10.1002/pd.1272

10. Lin LH, Maestá I, Braga A, Sun SY, Fushida K, Francisco RPV, et al. Multiple pregnancies with complete mole and coexisting normal fetus in North and South America: A retrospective multicenter cohort and literature review. Gynecol Oncol. 2017;145(1):88-95. https://doi.org/10.1016/j.ygyno.2017.01.021

11. Fowler DJ, Lindsay I, Seckl MJ, Sebire NJ. Routine pre-evacuation ultrasound diagnosis of hydatidiform mole: Experience of more than 1000 cases from a regional referral center. Ultrasound Obstet Gynecol. 2006;27(1):56-60. https://doi.org/10.1002/uog.2592

12. Bruchim I, Kidron D, Amiel A, Altaras M, Fejgin MD. Complete hydatidiform mole and a coexistent viable fetus: report of two cases and review of the literature. Gynecol Oncol. 2000;77(1):197-202. https://doi. org/10.1006/gyno.2000.5733

13. Jones WB, Lauersen NH. Hydatidiform mole with coexistent fetus. Am J Obstet Gynecol. 1975;122(3):26772. https://doi.org/10.1016/0002-9378(75)90165-9
14. Sebire NJ, Foskett M, Paradinas FJ, Fisher RA, Francis RJ, Short D, et al. Outcome of twin pregnancies with complete hydatidiform mole and healthy cotwin. Lancet. 2002;359(9324):2165-6. https://doi. org/10.1016/S0140-6736(02)09085-2

15. Vélez-Álvarez GA, Agudelo-Jaramillo B, Gómez-Dávila JG, Zuleta-Tobón JJ. Código rojo: Guía para el manejo de la hemorragia obstétrica. Rev Colomb Obstet Ginecol. 2009;60(1):34-48. https://doi.org/10.18597/rcog.352

16. Block MF, Merrill JA. Hydatidiform mole with coexistent fetus. Obstet Gynecol. 1982;60(1):129-134.

17. Pool R, Lebethe SJ, Lancaster EJ. Partial hydatidiform mole with a coexistent live full-term fetus. A case report. S Afr Med J. 1989;75(4):186-7. PMID: 2919342 18. Sarno AP Jr, Moorman AJ, Kalousek DK. Partial molar pregnancy with fetal survival: an unusual example of confined placental mosaicism. Obstet Gynecol. 1993;82(4 Pt 2 Suppl):716-9. https://doi. org/10.1097/00006250-199310020-00031

19. Feinberg RF, Lockwood CJ, Salafia C, Hobbins JC. Sonographic diagnosis of a pregnancy with a diffuse hydatidiform mole and coexistent 46,XX fetus: A case report. Obstet Gynecol. 1988;72(3 Pt 2):485----8. PMID: 3043299

20. Hartfield VJ. Ptyalism and partial hydatidiform mole associated with a normal term male fetus. Aust N Z J Obstet Gynaecol. 1983;23(1):53-6. https://doi. org/10.1111/j.1479-828X.1983.tb00161.x

21. Crooij MJ, van der Harten JJ, Puyenbroek JI, van Geijn HP, Arts NF. A partial hydatidiform mole, dispersed throughout the placenta, coexisting with a normal living fetus. Case report. Br J Obstet Gynaecol. 1985;92(1):104-6. https://doi. org/10.1111/j.1471-0528.1985.tb01057.x

22. Deaton JL, Hoffman JS, Saal H, Allred C, Koulos JP. Molar pregnancy coexisting with a normal fetus: A case report. Gynecol Oncol. 1989;32(3):394-7. https://doi. org/10.1016/0090-8258(89)90649-5

23. Thomas EJ, Pryce WI, Maltby EL, Duncan SL. The prospective management of a coexistent hydatidiform mole and fetus. Aust N Z J Obstet Gynaecol. 
1987;27(4):343-5. https://doi.org/10.1111/j.1479828X.1987.tb01024.x

24. Cheung NY, Ngan HY, Ghosh A. Persistent gestational trophoblastic disease after a diploid partial hydatidiform mole coexisting with a normal living fetus. Int J Gynaecol Obstet. 1992;38(3):238-9. https://doi. org/10.1016/0020-7292(82)90137-0

25. Hsieh CC, Hsieh TT, Hsueh C, Kuo DM, Lo LM, Hung TH. Delivery of a severely anaemic fetus after partial molar pregnancy: Clinical and ultrasonographic findings. Hum Reprod. 1999;14(4):1122-6. https:// doi.org/10.1093/humrep/14.4.1122

26. Lembet A, Zorlu CG, Yalçin HR, Seçkin B, Ekici E. Partial hydatidiform mole with diploid karyotype in a live fetus. Int J Gynaecol Obstet. 2000;69(2):149-52. https://doi.org/10.1016/S0020-7292(00)00179-X

27. Parveen Z, Bashir R, Jadoon T, Qayum I. Partial hydatidiform mole along with term gestation and alive baby. J Ayub Med Coll Abbottabad. 2004;16(4):84-5. PMID: 15762073.

28. Guven ESG, Ozturk N, Deveci S, Hizli D, Kandemir O, Dilbaz S. Partial molar pregnancy and coexisting fetus with diploid karyotype. J Matern Fetal Neonatal Med. 2007;20(2):175-81. https://doi. org/10.1080/14767050601134991

29. Tamrakar SR, Chawla CD. Preterm gesta on along with par al hydatidiform mole and alive foetus. Kathmandu Univ Med J. 2011;35(3):222-4. https://doi. org/10.3126/kumj.v9i3.6310

30. Shobhau N, Dhnanjaya BY, Nanada SK, Gopal N, Tejeswini KK, Musarrat Y. A term pregnancy wiyh partial molar changes. A case report. Int J Biol Med Res. 2011;(2):11191-2.

31. Sak ME, Soydinc HE, Evsen MS, Sak S, Firat U. Diploid karyotype partial mole coexisting with live term fetus_-Case report and review of the world literature. Ginekol Pol. 2012;83(10):789-91. PMID: 23383567.

32. Rathod AD, Pajai SP, Gaddikeri A. Partial mole with coexisten viable fetus_-A clinical dilema: Case report and reviw of literatura. J South Asian Feder Obst
Gynae. 2014;6(1):51-5. https://doi.org/10.5005/jpjournals-10006-1270

33. Ara R, Begum J, Kasem SB, Hoque S, Nargis SF. Partial hydatidiform mole with alive term IUGR foetus. J Bangladesh Coll Phys Surg. 2017;34(3):164-7. https:// doi.org/10.3329/jbcps.v34i3.32350

34. Flórez C, Martínez D, Álvarez O, García E. Embarazo molar de 25 semanas con feto vivo. Reporte de autopsia. Rev Chil Obstet Gynecol. 2016;(81)6:502-6. https:// doi.org/10.4067/S0717-75262016000600008

35. Muciño-García E, Hinojosa-Rodríguez KA, López-Rioja MJ, Salgado-Benítez E, Pérez-Lara SA, Zebadúa-Jiménez VH, López-Recio Y. Embarazo molar con feto vivo y éxito perinatal. Reporte de un caso y revisión bibliográfica. Gynecol Obstet Mex. 2017; 85(11)722-77.

36. Atuk FA, Basuni JBM. Molar pregnancy with normal viable fetus presenting with severe pre-eclampsia: A case report. J Med Case Rep. 2018;12(1):140. https:// doi.org/10.1186/s13256-018-1689-9

37. Zeng C, Chen Y, Zhao L, Wan B. Partial hydatidiform mole and coexistent live fetus: A case report and review of the literature. Open Med (Wars). 2019;14:843-6. https://doi.org/10.1515/med-2019-0098

38. De Franciscis P, Schiattarella A, Labriola D, Tammaro C, Messalli EM, La Mantia E, et al. A partial molar pregnancy associated with a fetus with intrauterine growth restriction delivered at 31 weeks: A case report. J Med Case Rep. 2019;13(1):204. https://doi. org/10.1186/s13256-019-2150-4

39. Hassan SA, Akhtar A, Ud Deen Z, Khan M, Jamal S, Sohail S, et al. Sad fetus syndrome: Partial molar pregnancy with a live fetus. Cureus. 2018;10(8):e3175. https://doi.org/10.7759/cureus.317

40. Sauerbrei EE, Salem S, Fayle B. Coexistent hydatidiform mole and live fetus in the second trimester: An ultrasound study. Radiology. 1980;135(2):415-7. https://doi.org/10.1148/radiology. 135.2.7367637

41. Teng NN, Ballon SC. Partial hydatidiform mole with diploid karyotype: Report of three cases. Am J Obstet Gynecol. 1984;150(8):961-4. https://doi. org/10.1016/0002-9378(84)90391-0 
Revista Colombiana de Obstetricia y Ginecología Vol. 71 No. 3 • 2020

42. Juárez A, Durán M, Islas L, Martínez R. Mola parcial con feto vivo. Análisis clínico morfológico y genético. Rev Mex Pediatría. 2009;(76)5:212-4.
43. Dhingra KK, Gupta P, Saroha V, Akhila L, Khurana N. Partial hydatidiform mole with a full-term infant. Indian J Pathol Microbiol. 2009;52(4):590-1. https:// doi.org/10.4103/0377-4929.56137

Conflicto de intereses: ninguno declarado. 